\title{
Identifying and Engineering the Stacking Sequence in CVD Grown Few-layer MoS 2 via Aberration-corrected STEM
}

\author{
Aiming Yan ${ }^{1,2}$, Wei Chen ${ }^{3}$, Colin Ophus ${ }^{4}$, Jim Ciston ${ }^{4}$, Christian Humberto Merino ${ }^{1}$, Alex Zettl ${ }^{1,2,5}$ \\ ${ }^{1 .}$ Department of Physics, University of California, Berkeley, Berkley, CA, USA. \\ 2. Materials Sciences Division, Lawrence Berkeley National Laboratory, Berkley, CA, USA. \\ ${ }^{3 .}$ Department of Mechanical, Materials and Aerospace Engineering, Illinois Institute of Technology, \\ Chicago, IL, USA. \\ 4. National Center for Electron Microscopy, Molecular Foundry, Lawrence Berkeley National \\ Laboratory, Berkeley, CA, USA. \\ ${ }^{5}$ Kavli Energy NanoSciences Institute at the University of California, Berkeley and the Lawrence \\ Berkeley National Laboratory, Berkeley, CA, USA.
}

As a promising candidate for the next-generation electronics, large-scale single- and few-layer $\mathrm{MoS}_{2}$ grown by chemical vapor deposition (CVD) method is an important advancement towards the technological implementation of this material [1][2]. However, compared to exfoliated $\mathrm{MoS}_{2}, \mathrm{CVD}$ grown few-layer $\mathrm{MoS}_{2}$ often exhibits mixed stacking sequences. These different stacking sequences can significantly impact the electronic and optical properties of $\mathrm{MoS}_{2}$, and are presumably caused by the hightemperature growth condition of CVD method. Here, we report the preferred intrinsic stacking sequence in CVD grown few-layer $\mathrm{MoS}_{2}$ identified by annular-dark-field (ADF) imaging in an aberration-corrected scanning transmission electron microscope (AC-STEM) operated at an ultra-low voltage-50 keV. We then use a combined in-situ AC-STEM setup to study the stacking sequence evolution of few-layer $\mathrm{MoS}_{2}$ under synergistic thermal effect and electron irradiation to unravel the role of high temperature in the final stacking sequence.

To probe the intrinsic stacking sequence in CVD grown $\mathrm{MoS}_{2}$, low-voltage STEM imging is an ideal tool. Considering the threshold for $S$ vacancy formation in single-layer $\mathrm{MoS}_{2}-80 \mathrm{keV}$ [3], the low operation voltage-50 keV enables the extensive imaging of atomically thin $\mathrm{MoS}_{2}$ without introducing significant structural damage. Figures 1a-c show the experimentally obtained STEM ADF images of CVD grown 1-, 2-, and 3-layer $\mathrm{MoS}_{2}$. By comparing to simulated STEM ADF images based on known stacking sequences, we identify the structure for 1-layer $\mathrm{MoS}_{2}$ is $\mathrm{H}$ phase, and the stacking sequence for 2-layer $\mathrm{MoS}_{2}$ is $\mathrm{AA}^{\prime}$, corresponding to $2 \mathrm{H}$ phase. We find 3-layer $\mathrm{MoS}_{2}$ has a preferred stacking sequence of AA'B, which is a mixed phase of $2 \mathrm{H}$ and $3 \mathrm{R}$. First-principles calculations reveal that the mixed phase is among the most stable stacking sequences in 3-layer $\mathrm{MoS}_{2}$ (fig. 1d) [4].

To understand how the growth temperature affects the final stacking sequence in CVD grown $\mathrm{MoS}_{2}$, we study the stacking sequence evolultion in few-layer $\mathrm{MoS}_{2}$ while it is in-situ heated at different temperatures and imaged by a higher-voltage electron beam $(80 \mathrm{keV})$. In this experiment, we start with $\mathrm{AB}$ stacked bilayer $\mathrm{MoS}_{2}$, the $80 \mathrm{keV}$ electron beam knocks out one layer of $\mathrm{MoS}_{2}$ atom-by-atom and leaves the H-phase single-layer $\mathrm{MoS}_{2}$ behind. The evaporated Mo and $\mathrm{S}$ atoms redeposit onto the neighboring $\mathrm{AB}$-stacked bilayer $\mathrm{MoS}_{2}$ and form trilayer $\mathrm{MoS}_{2}$ with different stacking sequences at different temperatures (Figs. 2 a-f). We find redeposited trilayer $\mathrm{MoS}_{2}$ at $600^{\circ} \mathrm{C}$ shows a preferred stacking sequence $\mathrm{ABB}$ (corresponding to $3 \mathrm{R}$ phase, Fig. 2g), and a high-energy defective $\mathrm{ABC}$ ' stacking at $700^{\circ} \mathrm{C}$. Our study suggests that high temperatue plays an important role in determining the stacking sequence in synthesized few-layer $\mathrm{MoS}_{2}$. 
References:

[1] S. Najmaei et al., Nat. Mater. 12 (2013), p. 754.

[2] A. M. van der Zande et al., Nat. Mater. 12 (2013), p.554.

[3] H. P. Komsa et al., Phys. Rev. Lett. 109 (2012), p. 035503.

[4] A. Yan et al., Physical Review B 93 (2016), p. 41420.
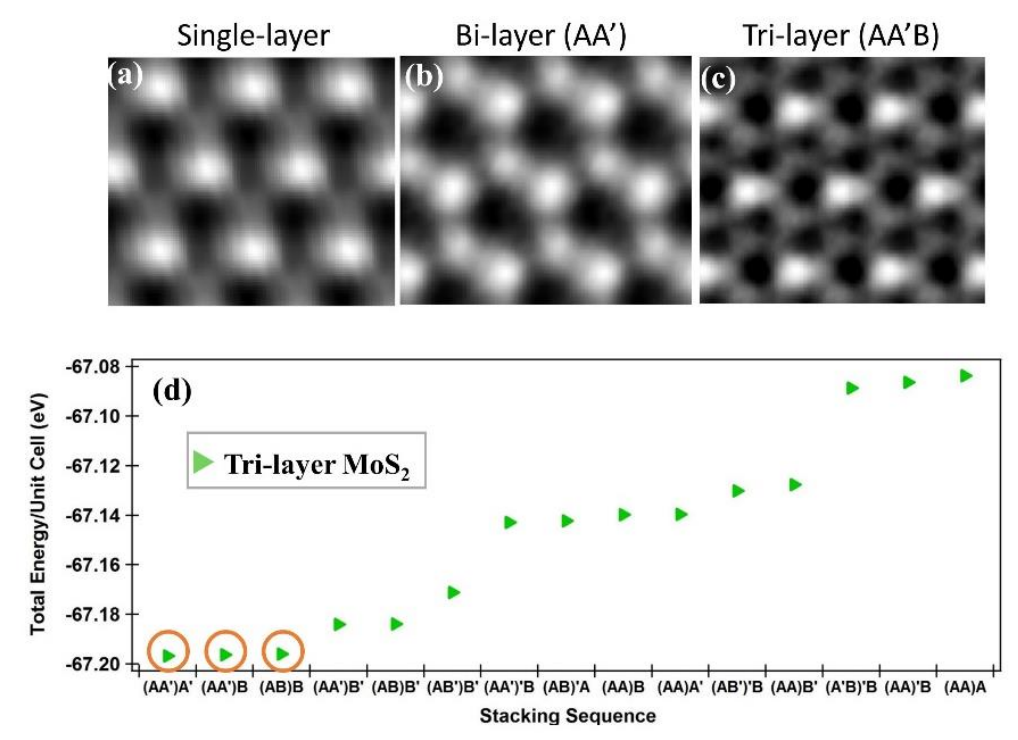

Figure 1. Experimental atomic-resolution STEM ADF images of single-layer ( $\mathrm{H}$ phase), bi-layer (AA' stacking) and tri-layer (AA'B stacking) CVD grown $\mathrm{MoS}_{2}$ (a-c, respectively), and stability of different stacking sequences in tri-layer $\mathrm{MoS}_{2}$ based on first-principles calculations (d).
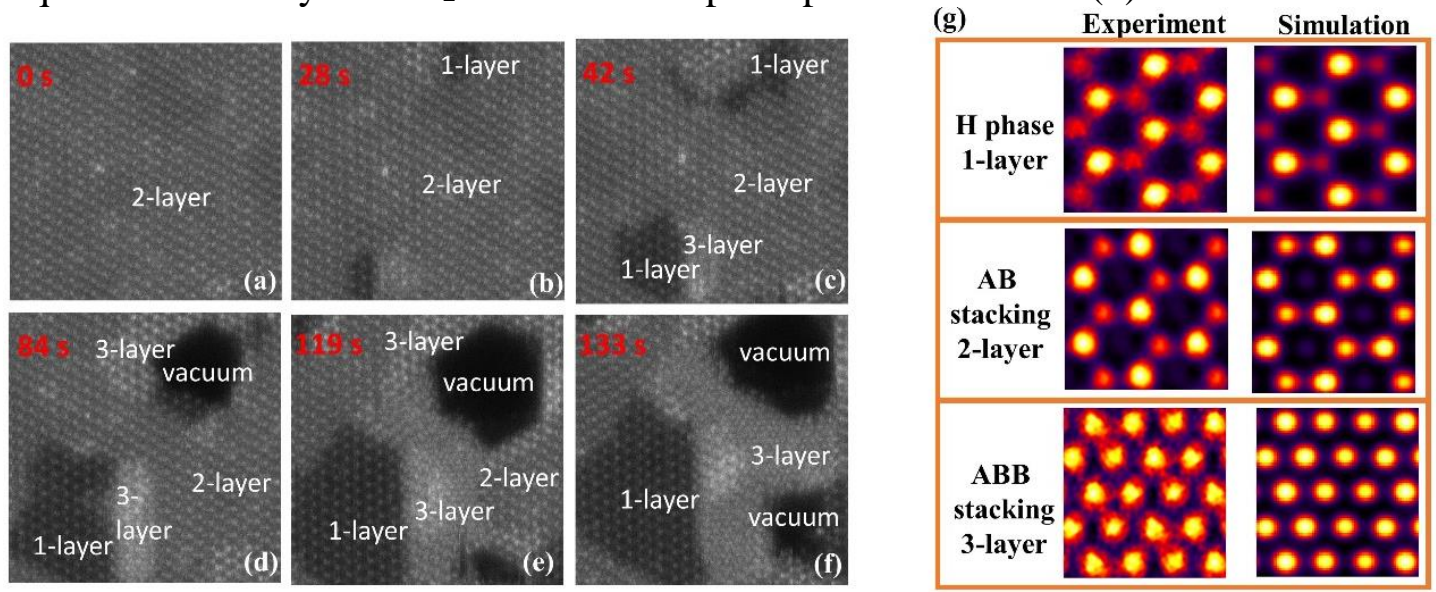

Figure 2. The evolution of stacking sequence in few-layer $\mathrm{MoS}_{2}$ while in-situ heated at $600{ }^{\circ} \mathrm{C}$ inside STEM. (a)-(f), Time-series STEM ADF images of bilayer $\mathrm{MoS}_{2}$ under the synergistic thermal effect and irradiation. Redeposited trilayer $\mathrm{MoS}_{2}$ shows a preferred stacking sequence $\mathrm{ABB}$, which corresponds to 3R phase. (g) identifying the stacking sequence in 1-, 2- and 3-layer $\mathrm{MoS}_{2}$ by comparison between the experimental and simulated STEM ADF images. 